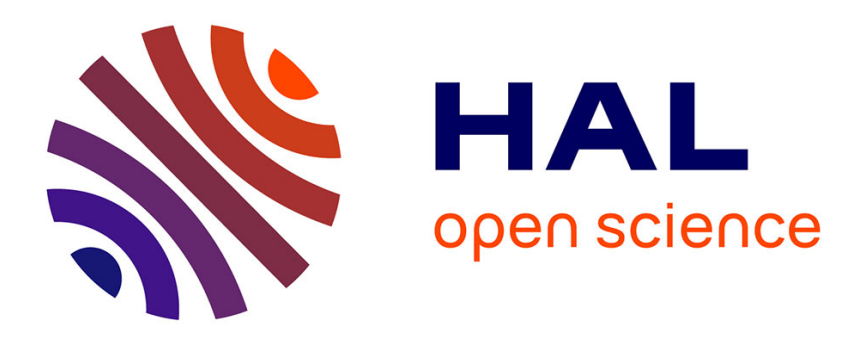

\title{
Improving diagnosis and management of primary brain tumors
}

Jean-Yves Delattre

\section{To cite this version:}

Jean-Yves Delattre. Improving diagnosis and management of primary brain tumors. Current Opinion in Neurology, 2017, 30 (6), pp.639-642. 10.1097/WCO.0000000000000500 . hal-01677369

\section{HAL Id: hal-01677369 \\ https://hal.sorbonne-universite.fr/hal-01677369}

Submitted on 8 Jan 2018

HAL is a multi-disciplinary open access archive for the deposit and dissemination of scientific research documents, whether they are published or not. The documents may come from teaching and research institutions in France or abroad, or from public or private research centers.
L'archive ouverte pluridisciplinaire HAL, est destinée au dépôt et à la diffusion de documents scientifiques de niveau recherche, publiés ou non, émanant des établissements d'enseignement et de recherche français ou étrangers, des laboratoires publics ou privés. 


\title{
Improving diagnosis and management of primary brain tumors
}

\author{
Jean-Yves Delattre ${ }^{\mathrm{a}, \mathrm{b}}$
}

Improving the diagnosis and management of gliomas and other primary brain tumors such as primary central nervous system (CNS) lymphoma (PCNSL) are clearly the key challenges of the neuro-oncology specialty. In the present section of Current Opinion in Neurology, leading experts update our knowledge on these issues and underline their practical significance.

For many years, neuro-oncologists have had great difficulties with the WHO classification of primary brain tumors. This classification was poorly reproducible between centers and even within one given center. Moreover, even when a pathologic consensus was obtained, neuro-oncologists were often puzzled by the heterogeneous course of patients within one given histologic entity. This was obvious for non-glioblastoma (GBM) diffuse gliomas but sometimes also in GBM patients. As a consequence, it was uneasy for the clinician to share accurate prognosis information with the patients and their families, to select the most appropriate treatment, and to avoid severe delayed toxicity when the course, unusually long, was different from expected. This heterogeneity also affected the analysis of clinical trials as patients with radically different courses, and likely different diseases, were included in the same trials. In this setting, the review by DeWitt et al. (pp. 643-649) of the new 2016 WHO classification is welcome and illustrates a major step forward for our discipline.

For the first time, the new WHO classification of CNS tumors 'integrates' histological and molecular diagnosis. In the large group of diffuse gliomas, the search for isocitrate deshydrogenase (IDH) mutation (and $1 \mathrm{p}-19 \mathrm{q}$ codeletion if IDH mutated) is now required to make an integrated diagnosis. It is important to realize that molecular information prevails. Thus, a tumor harboring an $I D H$ mutation and a $1 \mathrm{p}-19 \mathrm{q}$ codeletion will have an integrated diagnosis of oligodendroglioma (IDH mutated, 1p$19 \mathrm{q}$ codeleted) even if the histological analysis suggests an astrocytoma (with or without an oligodendroglial component). This conceptual change has major consequences for the clinician's practice as there is ample evidence that adding molecular diagnosis identifies much more homogeneous group of patients and correlates better with outcome than did classical histopathology. For example, retrospective reclassification of one of the largest trials dedicated to anaplastic oligodendroglial tumors (EORTC phase III trial 26951) [1] reveals that only $40 \%$ of tumors included in this trial would be classified today as oligodendroglioma using the integrated classification, whereas the remaining $60 \%$ would be classified as astrocytoma or GBM, and indeed they carried a much worse prognosis. In such an heterogeneous setting, it is quite fortunate that subgroup analysis eventually had enough power to detect the favorable impact of chemotherapy in what is called today an oligodendroglioma (IDH mutant, 1p-19q codeleted) [2]. For the clinician, the new classification has some downside. First, molecular diagnosis should be obtained in due time, before the onset of postsurgical treatment, which requires the need of adequate logistical support in the center. Second, the new classification leads to an increased use of the term 'not otherwise specified', which mainly designates tumors that cannot be classified into a precise molecular subgroup. In these patients, management decision has to be taken without the support of firmly established standards.

An important and still unsettled point is the interest of grading diffuse gliomas into grades II, III, or IV when an integrative diagnosis is made. Data in $I D H$-mutated astrocytoma suggest that grading does not really matter in this molecularly defined subtype. In oligodendrogliomas (IDH mutated, 1p19q codeleted), it is unknown whether grade II and grade III tumors share a common prognosis and require similar management. Until recently, one was considered a 'low grade glioma' and the other

\footnotetext{
${ }^{a}$ AP-HP, Hôpitaux Universitaires La Pitié Salpêtrière - Charles Foix, Service de Neurologie 2-Mazarin and ${ }^{\mathrm{b}}$ Inserm U 1127, CNRS UMR 7225, Sorbonne Universités, UPMC Univ Paris 06 UMR S 1127, Institut du Cerveau et de la Moelle épinière, ICM, Paris, France

Correspondence to Jean-Yves Delattre, MD, Service de Neurologie Mazarin, Hôpital de la Salpêtrière, Paris, France. Tel: +33 1421603 85; e-mail: jean-yves.delattre@aphp.fr
} 
a 'malignant anaplastic' tumor. If it is firmly demonstrated that grading is not an important factor as compared with molecular characterization in diffuse gliomas, this will have an important impact on patient management and design of clinical trials, as grade II and III patients will be eligible for the same treatments and trials.

Another issue is the speed of discovery of new molecular alterations with clinical relevance in recent years. It has been so rapid that the fear has been expressed that the WHO classification could always be 'one war late'. For this reason, as presented by DeWitt et al., (pp. 643-649) new tools such as 'cimpact now' (Consortium to Inform Molecular and Practical Approaches to CNS Tumor Taxonomy) accessible through website are being developed to provide real-time updates and publish clinically useful consensus recommendations more rapidly.

It is often stated in the introduction of articles on this subject that 'the management and prognosis of gliomas did not significantly change over the last decades'. This is not the reality. Although gliomas remain incurable tumors, important advances have been made in the management of non-GBM gliomas, particularly IDH mutated, which constitute, as a whole, more than two-thirds of non-GBM diffuse gliomas. In these patients, the important benefit of adding chemotherapy (Procarbazine-CCNU-Vincristine combination or Temozolomide) to radiotherapy has been clearly shown [3-5]. Survival exceeding 20 years is no longer an exception in oligodendroglioma (1p-19q codeleted) with two practical consequences: every effort should be made to reduce treatment toxicity in these patients, particularly the delayed cognitive impact of radiotherapy. Considerable patience will be needed to interpret the results of ongoing trials since previous experience has shown that premature conclusion of clinical trials could be misleading.

In GBM (IDH wild type), therapeutic advances have been real (Temozolomide in the 'Stupp regimen', and more recently tumor treating fields) [6], also demonstrated in the frail and elderly population [7]. However, the benefit remains modest and there is indeed an urgent need to develop new treatments of GBM.

Recent efforts have been directed in two main directions: first, precision therapy targeting specifically key molecular alterations/pathways of the tumors and second, immunotherapy.

Extensive molecular characterization of GBM and identification of several of the key pathways involved in their oncogenesis, combined with the design of specific agents targeting these pathways, have led to the hope that precision therapy was within reach. To date, targeted therapy, mainly with single agents, has been disappointing in GBM even if this approach appears to benefit to some rare forms of non-GBM gliomas (e.g., Everolimus in subependymal giant cell astrocytoma associated with tuberous sclerosis complex [8] or Vemurafenib/Dabrafenib in pleomorhic xanthoastrocytoma with BRAF V600E mutation [9]). Lack of efficacy of targeted therapy in GBM appears multifactorial (poor drug delivery, intratumoral heterogeneity, upregulation of redundant pathways, or inadequate design of clinical trial). Clearly, much tenacious work remains to be done to overcome these issues, including better preclinical selection of potential agents or their combinations on relevant models before clinical application.

The recent success of immunotherapy in various tumors has raised considerable hope that this approach could also be of help in primary brain tumors, particularly gliomas. Mildenberger et al. (pp. 650658) thoroughly review this important topic. They emphasize the specific immunologic aspects of gliomas, which constitute, with their microenvironment, a strikingly immunosuppressed area.

Immune checkpoints such as cytotoxic $\mathrm{T}$ lymphocyte-associated antigen 4, the programmed death-1 receptor, or its ligand (PD-L1) are inhibitory receptors found on immune cells (and also some normal and tumor cells) that, when stimulated, cause those immune cells to become quiescent. Antibodies blocking the stimulation of these negative checkpoints (immune checkpoint inhibitors, ICIs) such as Ipilimumab, Nivolumab or Pembrolizumab allow immune cells to remain activated and undergo effector function against cancer cells. Important efficacy in some systemic cancers (melanoma, nonsmall cell lung cancer), as well as the finding of expression of PD-L1 in gliomas, prompted their evaluation in GBM. On the contrary, results recently reported in the phase III study of recurrent GBM (CheckMate 143) did not show a benefit of Nivolumab as compared with Bevacizumab monotherapy [10]. In newly diagnosed GBM (with or without O(6)-MethylguanineDNA methyltransferase promoter methylation), two randomized trials evaluating the addition of Nivolumab to conventional treatment are ongoing. Mildenberger et al. (pp. 650-658) point out the limitations of checkpoint inhibitions in GBM in which several factors, including relatively low mutational load and neoepitopes, constitute negative predictive factor of response [11]. The situation could be different in recurrent low-grade gliomas, because the relatively slow growth of the tumor allows the accumulation of mutations, particularly in some patients pretreated with Temozolomide (hypermutator phenotype) and could also give enough time for an endogenous T-cell response to become effective. 
Vaccination is an important field and many studies are ongoing. However, the recently reported negative results of a randomized phase III trial evaluating Rindopepimut: Celldex Therapeutics, Inc, Hampton, NJ, USA, a peptide vaccine that targets epidermal growth factor receptor variant III (EGFRvIII) together with conventional treatment in patients with newly diagnosed GBM and harboring the EGFRvIII mutation (ACT-IV) was a disappointment [12]. Intratumoral heterogeneity has been incriminated in this failure and targeting true driver mutations, present in all tumor cells, appears critical. In this perspective, targeting mutated IDH1 as a neoepitope is an important ongoing axis of research.

As stated by the authors, the use of genetically modified $\mathrm{T}$ cells is a new avenue to develop immunotherapy of gliomas and early work using T cells expressing chimeric antigens (Chimeric antigen receptors T cells) is promising. Thus, despite some recent disappointments with vaccines and ICI, the field of immunotherapy in gliomas remains extremely active. As for targeted therapy, it will require very careful planning of future clinical trials.

Immune checkpoints constitute a natural mechanism to prevent excessive inflammatory damage and autoimmunity in healthy tissues, and it is therefore not surprising that their inhibition may lead to serious immunotoxicity and autoimmunity. As the use of ICIs in oncology has become widespread, it is important for neurologists to be aware of this new chapter among the vast field of complications of cancer treatment. The neurologic complication of ICI and their management are carefully reviewed by Touat et al. (pp. 659-668) Although neurologic complications of ICI are rare in comparison with other systemic toxicities (dermatologic, gastrointestinal, hepatic, and endocrine toxicities), they are important to recognize early, because they may be life threatening, raise complex differential diagnosis questions, and require urgent treatment. In the CNS, encephalitis is the most characteristic neurologic complications occurring without or with well characterized autoantibodies (such as N-methyl-D-aspartate receptor or Contactin Associated Protein 2 antibodies). In the peripheral nervous system, Guillain-Barré like syndromes may occur but the most paradigmatic complication is myasthenic syndrome, sometimes associated with necrotizing myositis. Neurologic complications generally respond well to corticosteroids but other immune-modulating treatments may be needed. Permanent discontinuation of ICI is needed when one face a life-threatening complication. In other cases, the decision to definitely discontinue ICI relies on careful multidisciplinary discussion, thoroughly weighing the risk/benefit ratio. As stated by the authors, a currently unsolved question is whether treatments with ICI can be given safely to patients with history of autoimmune neurological disorder [13]. Some data suggest increase risk of relapse/exacerbation in patients with a personal history of multiple sclerosis [14].

Though infrequent, all departments of neurooncology and all neurologists regularly see immunecompetent patients suffering from PCNSL.

Royer-Perron et al. (pp. 669-676) review and update our knowledge on this topic.

Although the reason of this singular CNS tropism remains unknown, some molecular alterations such as mutation in MYD88 and CD79B are more frequent in PCNSL that in their systemic counterpart though they share a common activation of the nuclear factor-kappa B (NF-кB) signaling pathway.

Of great potential interest for diagnostic and follow-up is the identification of biomarkers in the blood or CSF of PCNSL. The best known marker is CSF level of IL10 which appears to be quite sensitive and specific at concentration over $4 \mathrm{pg} / \mathrm{ml}$. Moreover, detection of MYD88 mutation or specific profiles of microRNA in circulating tumor cells or circulating tumor DNA in blood or CSF of PCNSL could soon reach clinical application, making PCNSL the first CNS tumor in which 'liquid biopsies' would be used. As the pioneer work of memorial sloan kettering cancer center group, important advances in the management of PCNSL patients have also been made. Overall median survival remain frustrating, barely exceeding two years, but it is worth noting that up to $20-25 \%$ of patients are now believed to be cured. Methotrexate still is the cornerstone of treatment while radiotherapy has been abandoned in the elderly (at least at conventional dose), and its role in younger patients is the subject of debate. As stated by the authors, an issue is to know whether high-dose chemotherapy with autologous stem cell transplantation could be an alternative to whole brain radiotherapy cancer center.

Among primary brain tumors, PCNSL is the tumor in which new therapeutic strategies appear most promising in a near future. Targeted therapy with ibrutinib (an inhibitor of Bruton's tyrosine kinase involved in the NF- $\mathrm{B}$ pathway) produced an impressive response rate in the recurrent setting $[15,16]$. Studies are starting to evaluate this agent in newly diagnosed PCNSL and in combination with chemotherapy. ICI also appears promising as well as other immunomodulatory agents derived from thalidomide (lenalidomide and pomalidomide) making PCNSL a very active field of therapeutic research in neuro-oncology.

In conclusion, neuro-oncology is now well structured worldwide, using standardized evaluation tools $[17,18]$ with active intergroups cooperation. Multidisciplinary cancer meeting, now enriched by 
molecular tumor board, constitutes the main pillar for appropriate decision making. In a near future, this multidisciplinary approach will benefit from the ongoing development of new diagnosis strategies such as radiomics, integrating imaging characteristics and genomic data, to refine treatment choice [19]. Addressing patients to clinical trials is critical but major efforts are needed to better select innovating agents at the preclinical level and to carefully design selective clinical trials to evaluate the full potential of targeted-therapies and immune-therapies.

\section{Acknowledgements}

None.

\section{Financial support and sponsorship}

None.

\section{Conflicts of interest}

There are no conflicts of interest.

\section{REFERENCES}

1. Dubbink HJ, Atmodimedjo PN, Kros JM, et al. Molecular classification of anaplastic oligodendroglioma using next-generation sequencing: a report of the prospective randomized EORTC Brain Tumor Group 26951 phase III trial. Neuro Oncol 2016; 18:388-400.

2. Van Den Bent MJ, Brandes AA, Taphoorn MJ, et al. Adjuvant Procarbazine, lomustine, and vincristine chemotherapy in newly diagnosed anaplastic oligodendroglioma: long-term follow-up of EORTC brain tumor group study 26951. J Clin Oncol 2013; 31:344-350.

3. Buckner JC, Shaw EG, Pugh SL, et al. Radiation plus procarbazine, CCNU, and vincristine in low-grade glioma. N Engl J Med 2016; 374:1344-1355.
4. Cairncross JG, Wang M, Jenkins RB, et al. Benefit from Procarbazine, Lomustine, and Vincristine in oligodendroglial tumors is associated with mutation of IDH. J Clin Oncol 2014; 32:783-790.

5. Chang S, Zhang P, Cairncross JG, et al. Phase III randomized study of radiation and Temozolomide versus radiation and nitrosourea therapy for anaplastic astrocytoma: results of NRG Oncology RTOG 9813. Neuro Oncol 2017; 19:252-258.

6. Stupp R, Idbaih A, Steinberg DM, et al. LTBK-01: prospective, multicenter phase III trial of tumor treating fields together with Temozolomide compared to Temozolomide alone in patients with newly diagnosed glioblastoma. Neuro Oncol 2016; 18(Suppl 6); https://doi.org/10.1093/neuonc/now260.

7. Perry JR, Laperriere N, O'Callaghan CJ, et al. Short-course radiation plus temozolomide in elderly patients with glioblastoma. N Engl J Med 2017; 376:1027-1037.

8. Curatolo P, Bjornvold M, Dill PE, et al. The Role of mTOR Inhibitors in the Treatment of Patients with Tuberous Sclerosis Complex: Evidence-based and Expert Opinions. Drugs 2016; 76:551-565.

9. Migliorini D, Aguiar D, Vargas Ml, et al. BRAF/MEK double blockade in refractory anaplastic pleomorphic xanthoastrocytoma. Neurology 2017; 88:1291-1293.

10. Reardon DA, Omuro A, Brandes AA, et al. Randomized phase 3 study evaluating the efficacy and safety of Nivolumab vs Bevacizumab in patients with recurrent glioblastoma: CheckMate 143. Neuro Oncol 2017; 19:iii21.

11. Hodges TR, Ott M, Xiu J, et al. Mutational burden, immune checkpoint expression, and mismatch repair in glioma: implications for immune checkpoint immunotherapy. Neuro Oncol 2017; 19:1047-1057.

12. Weller M, Butowski $N$, Tran D, et al. ATIM-03 ACT IV: an international, double blind phase 3 trial of rindopepimut in newly diagnosed, EGFRvIll expressing glioblastoma Neuro-Oncol 2016; 18(Suppl_6):vi17-vi18.

13. Johnson DB, Sullivan RJ, Ott PA, et al. Ipilimumab therapy in patients with advanced melanoma and preexisting autoimmune disorders. JAMA Oncol 2016; 2:234-240.

14. Gerdes LA, Held K, Beltrán $\mathrm{E}$, et al. CTLA4 as immunological checkpoint in the development of multiple sclerosis. Ann Neurol 2016; 80:294-300.

15. Grommes $C$, Pastore $A$, Palaskas N, et al. Ibrutinib unmasks critical role of bruton tyrosine kinase in primary CNS lymphoma. Cancer Discov 2017; 7:1018-1029.

16. Chamoun $\mathrm{K}$, Choquet $\mathrm{S}$, Boyle $\mathrm{E}$, et al. Ibrutinib monotherapy in relapsed/ refractory CNS lymphoma: a retrospective case series. Neurology 2017; 88:101-102.

17. Wen PY, Chang SM, Van den Bent MJ, et al. Response assessment in neurooncology clinical trials. J Clin Oncol 2017; 35:2439-2449.

18. Nayak $L$, DeAngelis LM, Brandes AA, et al. The Neurologic Assessment in Neuro-Oncology (NANO) scale: a tool to assess neurologic function for integration into the Response Assessment in Neuro-Oncology (RANO) criteria. Neuro Oncol 2017; 19:625-635.

19. Hu LS, Ning S, Eschbacher JM, et al. Radiogenomics to characterize regional genetic heterogeneity in glioblastoma. Neuro Oncol 2017; 19:128-137. 